\title{
INVESTIGATION OF GEOMETRIC PERFORMANCE OF AN INDOOR MOBILE MAPPING SYSTEM
}

\author{
Mehdi Maboudi, Dávid Bánhidi, and Markus Gerke \\ Institute of Geodesy and Photogrammetry, Technical University of Brunswick (Braunschweig), Germany; (m.maboudi@tu-bs.de)
}

\author{
Commission II, WG II/7
}

KEY WORDS: Mobile Laser Scanning, Evaluation, Viametris iMS3D, Point Cloud, Indoor Modeling

\begin{abstract}
:
Up-to-date and reliable 3D information of indoor environments is a prerequisite for many location- based services. One possibility to capture the necessary 3D data is to make use of Mobile Mapping Systems (MMSs) which rely for instance on SLAM (simultaneous localization and mapping). In most indoor environments, MMSs are by far faster than classic static systems. Moreover, they might deliver the point clouds with higher degree of completeness. In this paper, the geometric quality of point clouds of a state-of-the-art MMS (Viametris iMS3D) is investigated. In order to quantify the quality of iMS3D MMS, four different evaluation strategies namely cloud to cloud, point to plane, target to target and model based evaluation are employed. We conclude that the measurement accuracies are better than $1 \mathrm{~cm}$ and the precision of the point clouds are better than $3 \mathrm{~cm}$ in our experiments. For indoor mapping applications with few centimeters accuracy, the system offers a very fast solution. Moreover, as a nature of the current SLAM-based approaches, trajectory loop should be closed, but in some practical situations, closing the local trajectory loop might not be always possible. Our observation reveals that performing continuous repeated scanning could decrease the destructive effect of local unclosed loops.
\end{abstract}

\section{INTRODUCTION}

Up-to-date and reliable 3D information of indoor environments is a prerequisite for many location- based services and applications such as Building Information Modeling (BIM), facility management, cultural heritage documentation, and post-hazard rescue management. In the industry sector, it is quite useful for machine tracking and materials transportation inside factories. Classic approaches such as using total stations and terrestrial laser scanners (TLS) might not be efficient for large and rapidly changing indoor environments. Utilizing these static approaches is cumbersome in indoor areas since a complete model requires high number of standpoints, while the appropriate line of sight and the quantity of tie points are hardly guaranteed. Furthermore, reliable GNSS signals are not available in most indoor areas. Therefore GNSS based localization is also not possible. Currently, the most promising approaches for these conditions are based on Mobile Mapping Systems which mainly rely on SLAM (simultaneous localization and mapping) algorithms and/or high-grade IMUs like the technology which is employed in Trimble indoor mobile mapping solutions (TIMMS).

Commercial indoor MMSs are delivered as trolley-based systems, backpack mobile systems or hand-held compact devices (Maboudi et al., 2017). Most of these systems are by far faster than classic devices. Furthermore, thanks to their mobile nature, the completeness of the generated point cloud can be much higher than the station-based static systems, which requires high-skilled operators to co-register the stations, accurately. These benefits are especially handy in complex and daily changing indoor environments. However, the mobility of the platform and subsequent quality of the SLAM output may decay the quality of the generated point cloud.
Different indoor MMSs with different innovative hardware and software are getting quite popular. Each of these systems may be suitable for some specific environments and/or applications. The prerequisite for the practical use of the MMS systems is to be aware of the quality of the provided data. While in some research the effect of different sensor parameters on the quality of the point cloud is investigated (Kersten et al., 2009; Soudarissanane et al., 2011), in this paper we aim at the overall quality assessment of the final point cloud acquired by a MMS namely Viametris iMS3D. In (Lehtola et al., 2017) the performance of eight state-of-the-art MMSs are compared against survey-grade Leica and Faro TLS point clouds captured from three distinct test sites, where each area had its own purpose to test different scenarios. The authors used full point clouds for evaluations. Proposing a metric for evaluation, they reported that two trolley based scanners i.e. FGI Slammer and the commercial NavVis outperform other MMSs in different sites of this research. Using a terrestrial laser scanner (Leica P20) as a reference, the quality of handheld GeoSLAM Zeb-Revo and the trolley based Viametris iMS3D in a relatively small test area is investigated in (Maboudi et al., 2017). Sirmacek et al. (2016) compared ZEB1 and Leica C10 TLS point clouds. For local patch fitting of the ZEB1 point cloud, standard deviation of less than a centimeter is reported and deviation of local normals from global normal of a wall were between 0-4 degrees. The capabilities of ZEB1 and Viametris iMMS are also discussed in (Thomson et al., 2013). Cloud to cloud distance and two model-fitting approaches are used to compare the point clouds generated by both systems with a reference point cloud acquired by a Faro Focus3D TLS. Recently, in (Tucci et al., 2018) point clouds which are acquired by three MMSs namely Kaarta Stencil, Leica Pegasus Backpack, and GeoSLAM Zeb-Revo are evaluated using a Z+F 5010C TLS point cloud as ground-truth. Three evaluation methods called cloud to cloud, point to point, and cloud to feature are used to compare the point clouds. 
In this paper, we investigate the performance of the iMS3D more comprehensively. We analyze the effect of different number of loops on the quality, and employ different methods to evaluate the quality, including cloud to cloud, point to plane, target to target and model-based evaluation. Moreover, on purpose we did not close the loop in one room by moving backwards. One aim is to analyze the effect of this real life situation on the (local) quality of the generated point cloud.

\section{MATERIALS AND METHODS}

\section{$2.1 \quad$ iMS3D}

Viametris iMS3D (Figure 1) is a trolley-based continuous 3D scanner which is able to capture more than 80,000 points per second and its detection range is between $10 \mathrm{~cm}$ and 30 meters. The instrument consists of three Hokuyo profile scanners, a built-in Ellipse2-A miniature Attitude and Heading Reference System (AHRS), and a FLIR Ladybug( ${ }^{\circledR}$ spherical camera rig. Two computers are also attached to the instrument; the processing unit a.k.a. slave PC which is responsible for the real-time SLAM calculation and data storage and a tablet with an installed Viametris measurement control software which provide an interface to monitor and manage the acquisition process (Viametris, 2017).

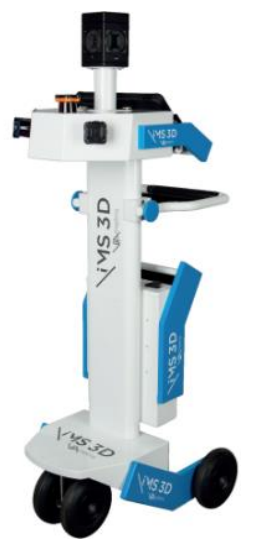

Figure 1. iMS3D (Viametris, 2017)

One of the Hokuyo laser profilers (UTM-30LX) is positioned horizontally to feed the SLAM algorithm and two lateral Hokuyo UTM-30LX-EW laser profilers which are positioned vertically, at a relative 90 degree angle at each other, in order to capture the main point cloud. The built-in Ellipse2-A miniature AHRS is a MEMS-based IMU which employing an extended Kalman filter provides roll and pitch data accurate to $0.2^{\circ}$ at $200 \mathrm{~Hz}$. Utilizing this sensor, unevenness of the surface (e.g. slopes or ramps) can be eliminated. Furthermore, the negative effect of quick rises like cables or small door steps can be mitigated (Leplomb, 2015). Sensor trajectory is also accessible which might be useful for some users. Ladybug3 spherical imaging system, which is mounted on the top of the instrument, consists of five cameras spread on a pentagon (side views) and one camera facing the ceiling for a full documentation of the environment. Pictures can be captured in every few meters automatically or manually.

According to the system provider, positioning accuracy of the system is $1 \mathrm{~cm}$ (Viametris, 2017) and Hokuyo laser profiler's accuracy is $3 \mathrm{~cm}$ in usual indoor distances (less than 10 meters). The iMS 3D $1 \mathrm{~cm}$ accuracy refers to the positioning of the system along the trajectory, which is obtained within the SLAM algorithm. Because of redundant observations, this accuracy is higher than single laser measurements.

\section{$2.2 \quad$ Test area:}

The test area is part of a building in the Technical University of Braunschweig, Germany, which consists of a long corridor, two rooms and some niches (Figure 2).

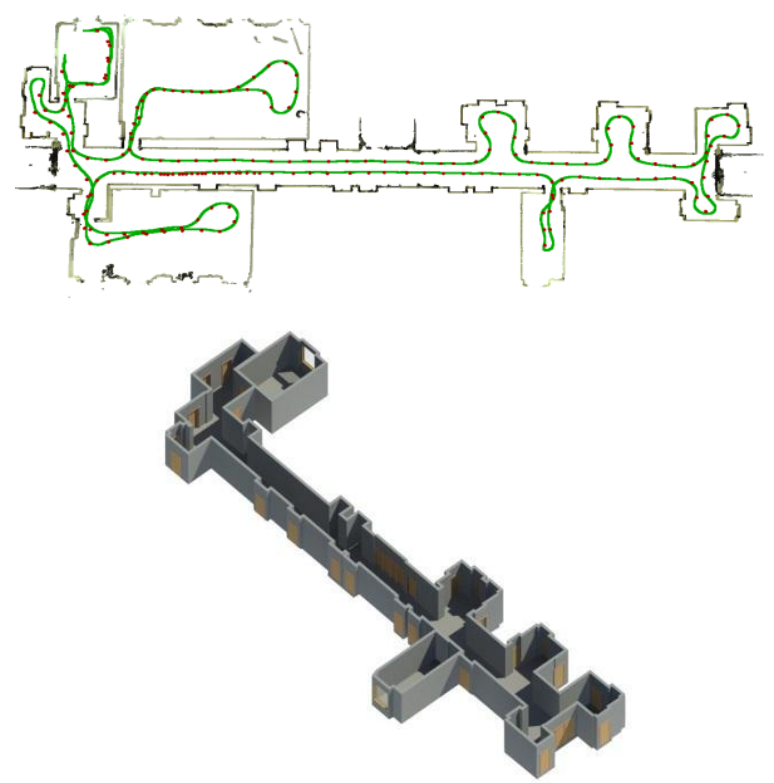

Figure 2. Test area; Top: iMS3D trajectory overlaid on 2D layout of the floor, bottom: 3D model of the test area.

The testing environment can be roughly described as a corridor with dimensions of approximately $39 \times 3 \times 4$ meters and two small rooms as shown in Figure 2. Two larger rooms visible in Figure 2, have also been captured but are not included in the reference dataset.

\subsection{Data capture strategy}

In order to quantify the repeatability of the point cloud acquisition with the iMS3D system, the scanning of the study area is repeated three times. For this purpose, we tried to keep the data capturing conditions unchanged, as much as possible. Moreover, in order to analyze the possible effects of repeated acquisition on point cloud quality, which is affected by the exploited SLAM algorithm, the scanning of the whole area is repeated in two and three continuous loops. Therefore, five distinct point clouds are provided from the same test area for further experiments. The following table lists general information about time for data acquisition, length of trajectory, number of registered points.

\begin{tabular}{|l|c|c|c|}
\hline Point Cloud & $\begin{array}{c}\text { Time } \\
\text { (minutes) }\end{array}$ & $\begin{array}{c}\text { Length of } \\
\text { Trajectory (m) }\end{array}$ & No. of Points \\
\hline 1loop 1st & $6: 34$ & $176 \mathrm{~m}$ & $33,9 \mathrm{M}$ \\
\hline 1loop 2nd & $6: 26$ & $188 \mathrm{~m}$ & $33,1 \mathrm{M}$ \\
\hline 1loop 3rd & $6: 16$ & $179 \mathrm{~m}$ & $32,3 \mathrm{M}$ \\
\hline 2loops & $12: 30$ & $351 \mathrm{~m}$ & $64,6 \mathrm{M}$ \\
\hline 3loops & $19: 28$ & $528 \mathrm{~m}$ & $94,5 \mathrm{M}$ \\
\hline
\end{tabular}

Table 1: General information about captured point clouds

Moreover, Leica P20 TLS and Leica Nova MS50 MultiStation are used alongside the iMS3D to provide enough information for the intended evaluation. Therefore, another point cloud of the test area and around 50 points (targets) are also added to our dataset. 
Another very useful and practical observation is an experiment that we designed in one of the rooms in the study area. Usually in small offices and rooms, which are equipped with different furniture and devices, it might not be practically possible to make a smooth and closed trajectory (Figure 3) which is highly advised by providers of many SLAM based MMSs. Therefore, in one of the rooms, we did not make a complete loop, but the operator entered the room and then moved backwards.
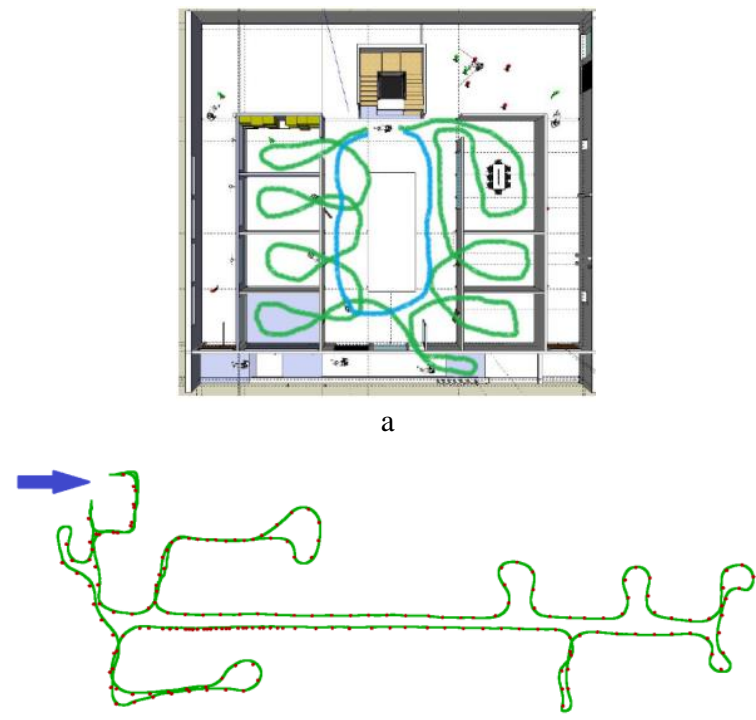

b

Figure 3. Sensor Trajectory; a) Ideal (closed loop) trajectory suggested by system provider (Viametris, 2017), and b) Intentional loop closure failure for simulating the possible practical situations.

\subsection{Targets}

Individual and easily identifiable targets that can be measured in any dataset for further quality analysis or comparison is a prerequisite of target to target evaluation (Maboudi et al., 2017; Tucci et al., 2018). For this purpose, we needed to choose an easily detectable but still accurately measurable target design. The first requirement can be accomplished by a large target size; however, for a precise measurement a smaller object is better. Therefore, we had to deal with this contradiction. In addition, the density of the point cloud is likely to be substantially different, so the number of point representing the target might vary in a wide range. We tested targets with different shapes and sizes; see an overview in Figure 4.
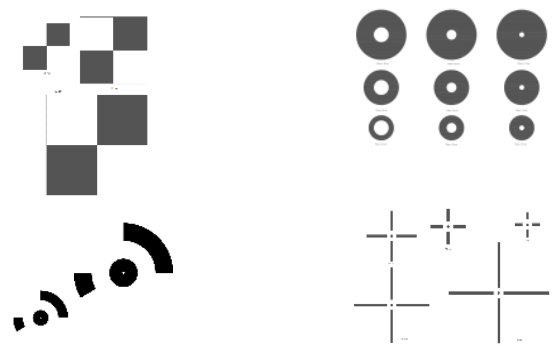

Figure 4. Different tested targets.

According to our observations, checkerboard-type targets (upper left part of the Figure 4) with the size about $10 \mathrm{~cm}$ provide the best compromise between detectability and pointing accuracy of the targets in the point clouds.

\subsection{Geometric evaluation:}

In order to investigate the quality of the generated point clouds, four different comparison approaches are utilized. Cloud to cloud distance, signed distance of the points to a corresponding plane (Nex et al., 2015), comparison of distance between some distinctive points (targets) and model based evaluation indicate the different aspects of geometric quality of the point clouds.

2.5.1 Cloud to cloud distance: Considering the P20 data as the reference, all iMS3D Point clouds are registered to the TLS point cloud using a six parameter similarity transform (3D- Helmert transformation with fixed scale), followed by iterative closest point (ICP) algorithm which is utilized for fine registration of the point clouds. Then, the cloud to cloud distances are computed using the CloudCompare $\mathrm{M} 3 \mathrm{C} 2$ plugin. For qualitative interpretation of the results, the signed cloud to cloud distances are depicted in Figure 5.

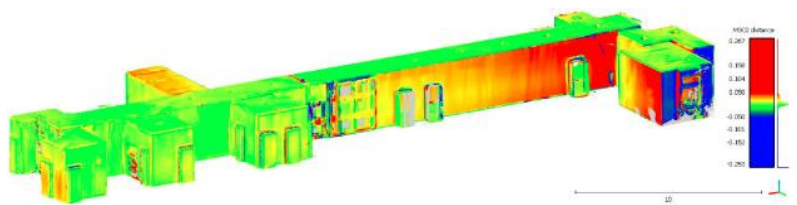

a

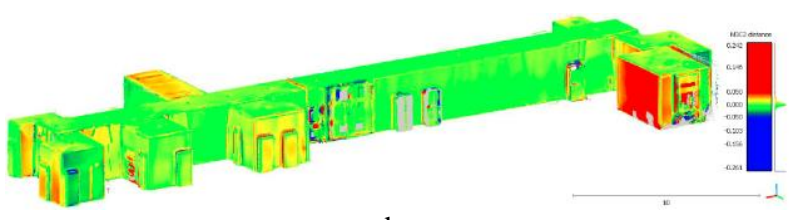

b

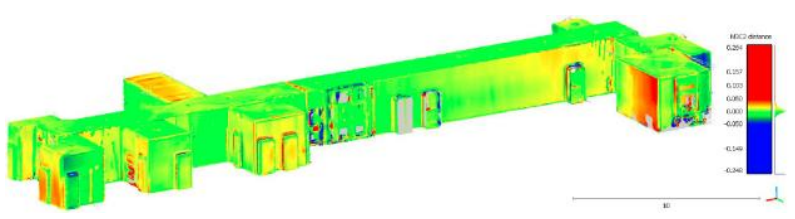

Figure 5. Cloud to cloud distances and the effect of increasing number of loops; a) one loop, b) two loops, and c) three loops.

As it can be seen in Figure 5, the effect of designed unclosed loop in a room in the right part of the test area is reflected in relatively large errors around that area. However, by increasing the number of loops, this effect is mitigated. One of the main outcomes of these experiments is that multiple scans of the same area using MLS system alleviate the local errors of the resulting point cloud. That is mainly on the account of the ability of the implemented SLAM of the system to find and benefit from more appropriate constraints for computing the adjusted trajectory of the sensor. For quantitative evaluation of the results, mean and standard deviation of the errors are computed and listed in Table 2.

\begin{tabular}{|l|c|c|}
\hline Point cloud & Mean $(\mathrm{mm})$ & Standard dev. $(\mathrm{mm})$ \\
\hline 1Loop 1st & 6.7 & 23.9 \\
\hline 1Loop 2nd & 6.7 & 22.5 \\
\hline 1Loop 3rd & 3.2 & 16.6 \\
\hline 2Loops & 4.4 & 16.0 \\
\hline 3Loops & 3.6 & 13.9 \\
\hline
\end{tabular}

Table 2: Cloud to cloud error analysis of point clouds 
All mean values in Table 2, are smaller than $1 \mathrm{~cm}$, the standard deviations are below $2,5 \mathrm{~cm}$; these numbers contain error from both systems (TLS and MMS). It is safe to say that all the errors, calculated with this method, are within the given error range. If we compare them against each other, almost all the time, the multiple loop data is superior. Furthermore, the 3 loops data is better than the 2loops data. The 1loop 3rd acquisition is obviously peeking from the other datasets. However, it is possibly just one fortunate occasion and the other two single loops' point clouds did not exhibit such a good performance, which is another indication for the necessity of the repeated scanning in environments with unavoidable unclosed loops.

2.5.2 Points to planes distance: Fitting planes to the points, which belong to a planar surface, can decrease the effect of reference instrument noise on the results. Therefore, we utilize the region growing approach introduced in (Vosselman and Maas, 2010) in order to detected planar surfaces in P20 TLS data. A total of 63 reliable planes are extracted. Next, planes are fitted to each group of these points using a least squares based approach. Afterwards, in order to facilitate a systematic analysis of the signed distance of the points with respect to the reference planes the normal vectors are computed and defined to point outward the room (Nex et al., 2015). Figure 6 illustrates the extracted planes and corresponding normal vectors.

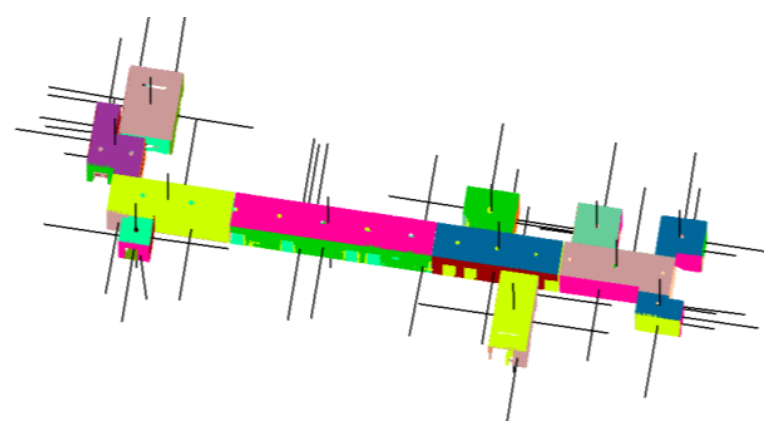

Figure 6. Detected reference planes and their normal vectors which point outward of the building.

There are two issues which should be taken into account during the computation of signed distances in indoor environments: 1- Most approaches use center of gravity (barycenter) for computing the direction of the normal vectors which is misleading for indoor point clouds of the buildings with non-convex outline shapes. One solution for this problem could be taking the sensor trajectory into account for computing the direction of normal. 2- Computing the signed distance to the indoor walls is also somehow meaningless for thin walls. Because, considering one plane for both sides of the wall affects the evaluation accuracy and directions of their normal vectors also depend on the viewing point in the indoor point clouds.

Considering these issues, for all five point clouds (cf. Table 1), the signed distance of all points assigned to each reference plane is computed. For this evaluation, we computed the histogram of the errors to check whether the errors follow a Normal distribution or not. It is also possible to use statistical tests like Q-Q plot (Höhle and Höhle, 2009; Nocerino et al., 2017 ) to investigate whether data originate from a normal distribution, but as stated in (Höhle and Höhle, 2009) these tests are often rather sensitive in case of large data sets. Therefore, we prefer the visual inspection of the distribution of errors.
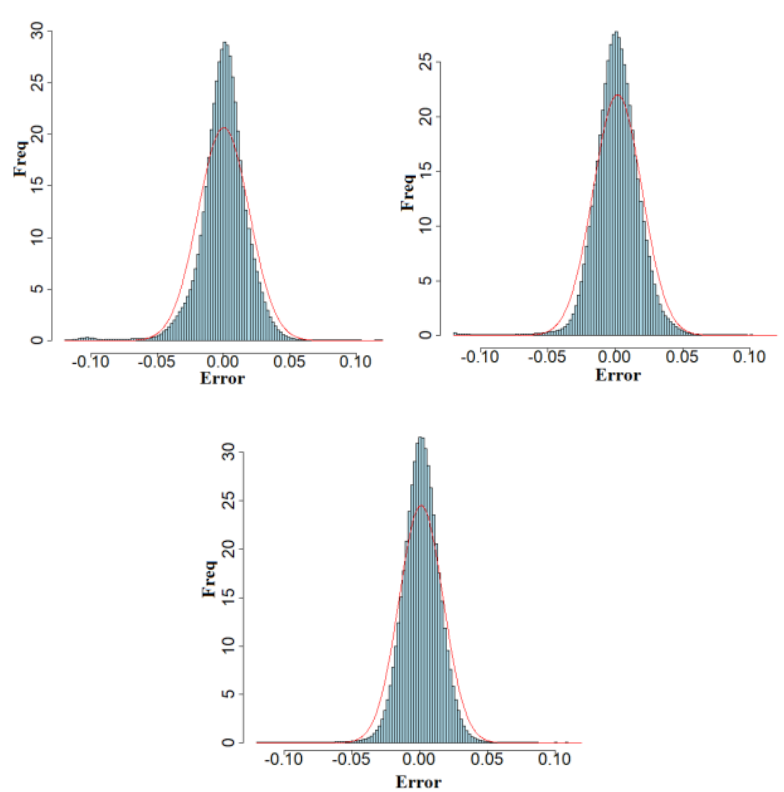

Figure 7. Distribution of point to plane errors. a) 1 Loop $3^{\text {rd }}$, b) 2Loops and c) 3Loops.

Our conclusion from the graphs in Figure 7 is that errors follow a bell-shaped distribution. However, normal standard deviation values are a bit pessimistic representation of the errors, since the actual values are better than what their normal distribution would suggest.

In the next step, mean and standard deviation of all signed distances in each point cloud are computed which are listed in Table 3. The first data row in Table 3 which is called TLS selftest reports the mean and standard deviation of the signed distances of the reference point cloud i.e. TLS point cloud to the reference planes. In other words, in order to verify the hypothesis about planarity of the walls and ceiling, all reference planes are evaluated using the same reference point cloud that is used before to calculate them. Obtained results verify that the reference fitted planes simplify the reference point cloud properly.

\begin{tabular}{|l|c|c|}
\hline Point cloud & Mean(mm) & Standard dev. (mm) \\
\hline TLS self-test & 0.1 & 3.6 \\
\hline 1Loop 1st & 2.2 & 25.2 \\
\hline 1Loop 2nd & 0.4 & 26.3 \\
\hline 1Loop 3rd & 0.1 & 19.3 \\
\hline 2Loops & 1.8 & 18.1 \\
\hline 3Loops & 1.6 & 16.3 \\
\hline
\end{tabular}

Table 3: Points to plane based error analysis of point clouds

Compared to the cloud to cloud statistics as shown in Table 2 some major differences are obvious for the mean values: while in the cloud to cloud experiment this value varies from 3 to $7 \mathrm{~mm}$, it is less than $2 \mathrm{~mm}$ for the point to plane data. Standard deviations are in the same range, but a bit worse for the pointto-plane data. The better mean values can be explained by the fact that during reference plane fitting random errors in the reference data are averaged out, hence, the distance values are only affected by errors in the test data. Unless there are nonparallel systematic shifts the mean distance should be around zero. 
2.5.3 Distance between some distinctive points (targets): In the third evaluation method, which is target to target distance calculation, 46 targets which were installed in different parts of the test area as depicted in Figure 8 are used.

Cloud to cloud and points to plane measures are somehow local measures, i.e. they are used to quantify the accuracy in the local vicinity. Global deformations, e.g. caused by an erroneous trajectory computation are difficult to identify with such local methods. One possibility to detect those effects is to compare the difference between corresponding distances within different datasets.

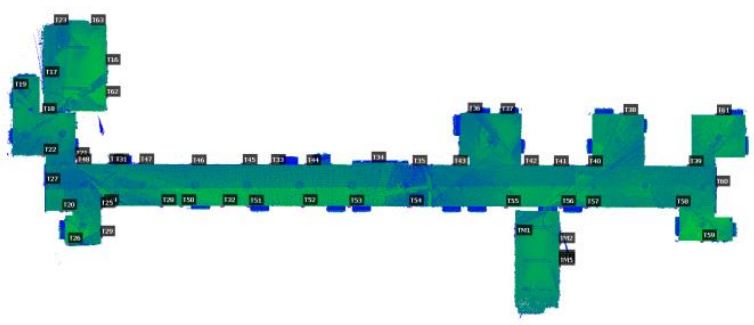

Figure 8. Distribution of targets in the test area.

Since this method requires distinctive points, all targets are measured in all 6 point clouds (5 iMS3D and the P20) and their 3D coordinates are used for evaluation. Furthermore, Leica MS50 multistation is also employed to measure the targets. Therefore, for each target we have seven 3D coordinates from P20, MS50 and different iMS3D data acquisitions.

For all datasets, 3D Euclidean distances between each target and all other targets are computed. Then, the difference between all distances and their corresponding reference distances are calculated. Then, these values which could be considered as errors are used for computing mean and standard deviation. Figure 9 shows these values in two different setups. In Figure 9a Leica P20 TLS data is considered as reference and in Figure 9b Leica MS50 targets are considered as reference.
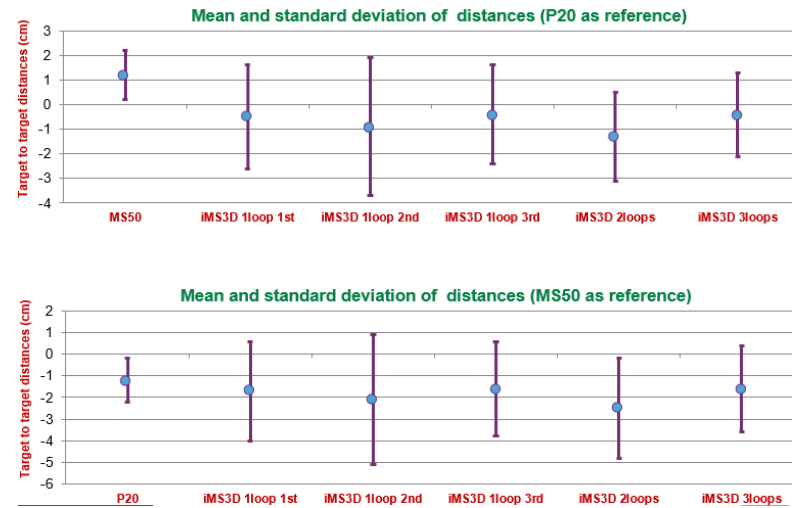

Figure 9. Target to Target evaluation; a) Leica P20 TLS as reference, b) Leica MS50 as reference.

For visual inspection of target to target evaluation a line-based comparison is performed where the targets are connected with lines which are colorized by the distance difference compared to the reference value. This way the local error of the targets can be disassociated to individual distance differences to track the source of the high error values. The result of the comparison between P20 and MS50 can be seen in Figure 10 .
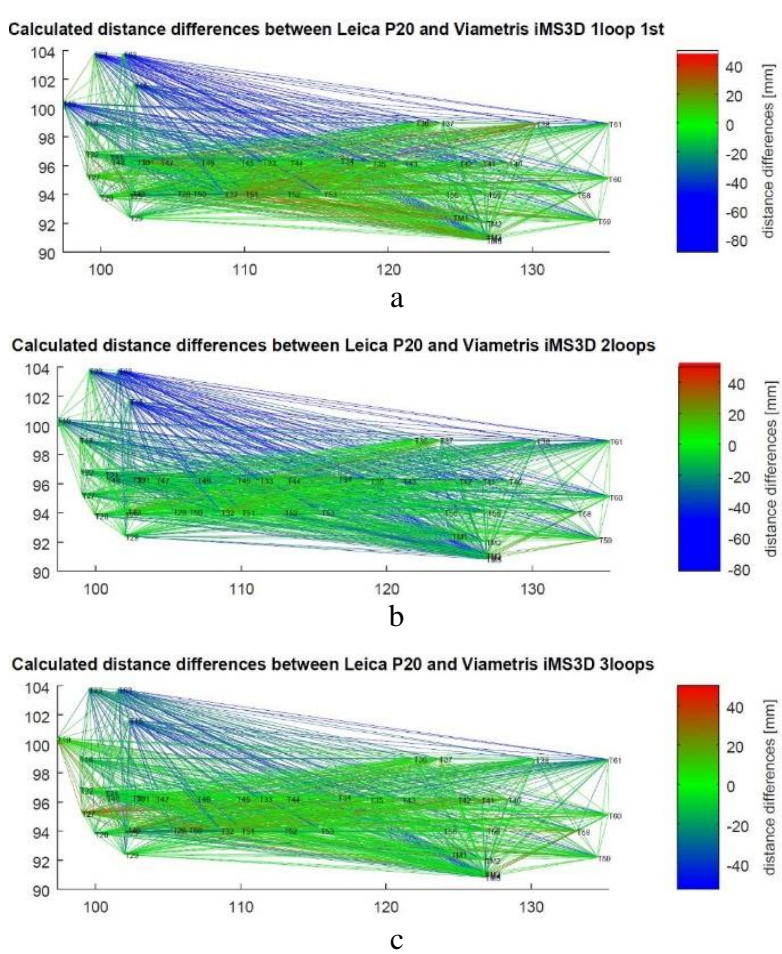

Figure 10. Effect of increasing number of loops; a) one loop, b) two loops, and c) three loops.

As it can be seen in Figure 10, the corridor area contains minimal errors, while the targets in the room with unclosed loop (upper left part of the image) show high differences. Almost all the lines connected to the problematic targets are blue, which means that these points (and the wall containing them) show a large misplacement.

Comparing Figure 10a, b, and $\mathrm{c}$ it is also evident that the effects of unclosed loop on error values decrease by increasing the number of loops. In order to better understand the obtained improvement, it should be accentuated that due to graphical considerations, the scale-bar in Figure 10c is different from others.

\subsubsection{Model-based evaluation}

In practical applications a $2 \mathrm{D} / 3 \mathrm{D}$ CAD map of the building is one of the most common outputs of the MMS point clouds. In model-based evaluation we directly generate CAD models from various point clouds and discrete measurements including wall thickness and room dimension are performed on different CAD models.

For the modeling, we used classical simple measuring method mostly used by architects i.e. disto measurement to generate a 2D map of the area. Next, PointCab software is used and an approximately half meter horizontal slice is segmented slightly under the upper part of the doorframe. This gives us a good representation of the position of the walls, doors and windows. Then by vectorization of the point clouds, the layout of the test area is extracted.

A 2D CAD model of the most complex part of the corridor is illustrated in Figure 11. Thickness of the walls and distance between opposite walls are compared by examining various models from different datasets. 


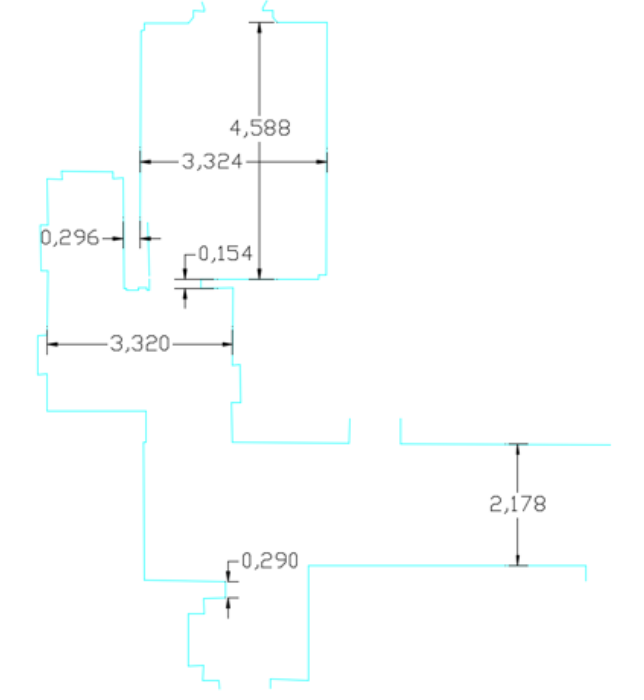

a

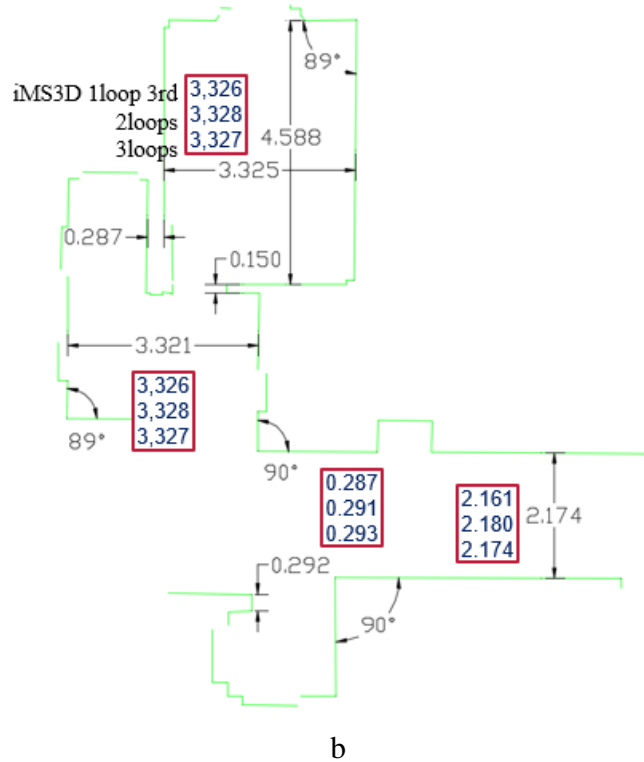

$\mathrm{b}$

Figure 11. Model based evaluation (a), Manual measurement data (b) Model using P20 as reference and overlaid iMS3D measurements (1 loop $3^{\text {rd }}, 2$ loops, 3 loops).

The results of this part show that iMS3D mobile mapping system is capable of delivering a good-enough model for usual architectural application, but is much faster than P20 TLS. However, for applications which need higher accuracy $(3 \mathrm{~cm}$ or better), TLS point clouds are still superior.

\section{CONCLUSION}

The system under investigation (Viametris iMS3D) belongs to cart-based indoor mobile mapping systems. Overall, the measurement accuracies are according to expectations (system provider accuracy i.e. $3 \mathrm{~cm}$ ). Each of four different comparison approaches gives the opportunity to investigate the system from a unique perspective and reveals some special characteristic of the provided point cloud. According to our observations, increasing the number of loops in data acquisition could mitigate the effect of local SLAM inaccuracy which was originated from an intentional unclosed loop in the trajectory. For indoor mapping applications with few centimeters accuracy, the system offers a very fast data acquisition, which can be converted to final colored point cloud after post-processing in the office. In our future experiments, we concentrate on improving computed system trajectory in non-ideal situations, extending the model- based evaluation and inspecting the repeatability and consistency of the generated point clouds by setting up some new data acquisitions.

\section{References}

Höhle, J. and Höhle, M., 2009. Accuracy assessment of digital elevation models by means of robust statistical methods, ISPRS Journal of Photogrammetry and Remote Sensing. Elsevier, 64(4), pp. 398-406.

Kersten, T. P., Mechelke, K., Lindstaedt, M. and Sternberg, H., 2009. Methods for Geometric Accuracy Investigations of Terrestrial Laser Scanning Systems, Photogrammetrie Fernerkundung - Geoinformation, 4, pp. 301-315.

Lehtola, V., Kaartinen, H., Nüchter, A., Kaijaluoto, R., Kukko, A., Litkey, P., Honkavaara, E., Rosnell, T., Vaaja, M., Virtanen, J.-P., Kurkela, M., El Issaoui, A., Zhu, L., Jaakkola, A. and Hyyppä, J., 2017. Comparison of the Selected StateOf-The-Art 3D Indoor Scanning and Point Cloud Generation Methods, Remote Sensing. MDPI, 9(8), p. 796.

Leplomb, H., 2015, Mobile Indoor 3D Scanner - Succes story, www.sbg-systems.com/docs/iMS-3D-Indoor-MobileScanner-Success-Story.pdf (12 October 2017).

Maboudi, M., Bánhidi, D. and Gerke, M., 2017, Evaluation of indoor mobile mapping systems, In: 20th Application-oriented Workshop on Measuring, Modeling, Processing and Analysis of 3D-Data, Berlin, Germany: GFaI, pp. 125-134.

Nex, F., Gerke, M., Remondino, F., Przybilla, H.-J., Bäumker, M. and Zurhorst, A., 2015, ISPRS Benchmark for Multiplatform Photogrammetry, in ISPRS Annals of Photogrammetry, Remote Sensing and Spatial Information Sciences. Munich, German, Volume II-3/W4, y, pp. 135-142.

Nocerino, E., Menna, F., Remondino, F., Toschi, I. and Rodríguez-Gonzálvez, P., 2017, Investigation of indoor and outdoor performance of two portable mobile mapping systems, In: SPIE 10332, Videometrics, Range Imaging, and Applications XIV,Munich, Germany, p. 103320I.

Soudarissanane, S. S., Lindenbergh, R., Menenti, M. and Teunissen, P., 2011, Scanning geometry: Influencing factor on the quality of terrestrial laser scanning points, ISPRS Journal of Photogrammetry and Remote Sensing, 66(4), pp. 389-399.

Thomson, C., Apostolopoulos, G., Backes, D. and Boehm, J., 2013, Mobile Laser Scanning for Indoor Modelling, ISPRS Annals of Photogrammetry, Remote Sensing and Spatial Information Sciences, Antalya, Turkey, Volume II-5/W2(2), pp. 289-293.

Tucci, G., Visintini, D., Bonora, V. and Parisi, E., 2018, Examination of Indoor Mobile Mapping Systems in a Diversified Internal/External Test Field, Applied Sciences. MDPI, 8(3), p. 401.

Viametris, C., 2017, iMS3D leaflet. www.viametris.com, (20 September 2017).

Vosselman, G. and Maas, H.-G. 2010, Airborne and terrestrial laser scanning. Whittles Publishing. 